\title{
Cost-Free Laser Caging in the Operating Room
}

\author{
Ryan A. Chandhoke, MD, Kamaljot S. Kaler, MD, and Ralph V. Clayman, MD
}

\begin{abstract}
We have assembled a three-step process for securing and presetting the functional length of the laser fiber to be used for ureteroscopic lithotripsy. For this process, three components are required: a sterile piece of adhesive, an empty guidewire casing with a pre-attached Luer-Lok ${ }^{\mathrm{TM}}$ fitting, and a SureSeal ${ }^{\circledR}$ adapter. The only additional piece of equipment for this arrangement not routinely used during ureteroscopy is the sterile adhesive strip.
\end{abstract}

K IDNEY STONES AFFECT $\sim 10 \%$ of the American population with evidence that the prevalence of urolithiasis is increasing in both men and women. ${ }^{1}$ A significant proportion of patients with stone disease require some form of surgical treatment. $^{2}$ For ureteral stones, ureteroscopy with holmium laser lithotripsy is associated with a greater stonefree rate and lower costs than shock wave lithotripsy and has become an accepted standard of care. ${ }^{3}$

A variety of holmium laser fibers are available for ureteroscopic lithotripsy. Some disposable holmium fibers are for a single use, whereas others may be refurbished and resterilized for multiple uses. Regardless, all holmium laser fibers need to be handled with care in the operating room to minimize possible fracture and exposure of laser light to

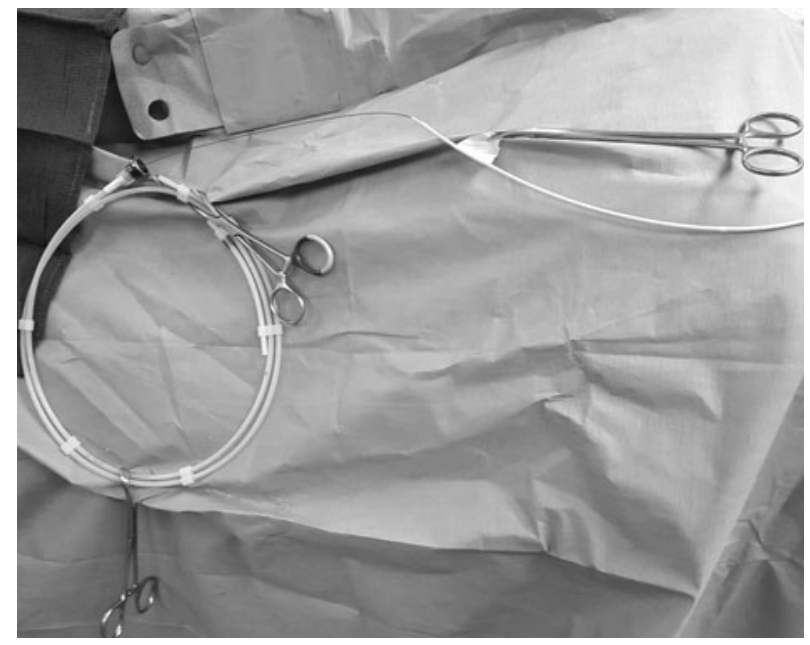

FIG. 1. Laser holder with all components including a sterile adhesive strip, an empty guidewire case (secured to the leg drape with two towel clamps), and a preattached Luer-Lok ${ }^{\mathrm{TM}}$ fitting connected to a SureSeal ${ }^{\circledR}$ adapter on the laser fiber.

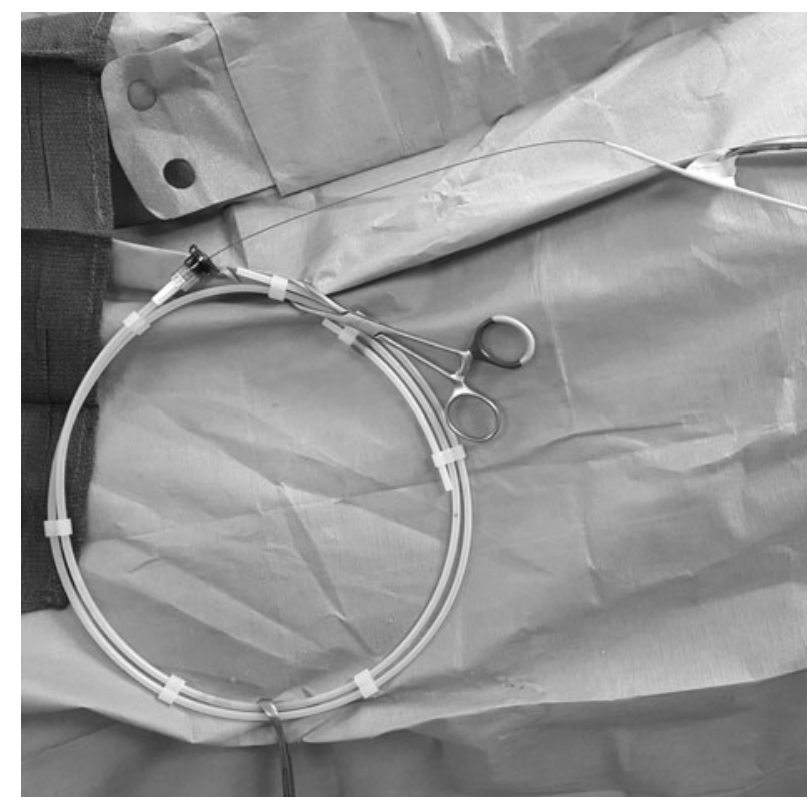

FIG. 2. A closer view of the laser holder secured to the leg drape.

the surgeon and operating room personnel. Also, during a procedure, the holmium laser fiber may need to be inserted and withdrawn multiple times, further risking damage to the fiber.

We have assembled a three-step process for securing and presetting the functional length of the laser fiber to be used for ureteroscopic lithotripsy (Figs. 1 and 2). For this process, three components are required: a sterile piece of adhesive (e.g., Steri-Strip ${ }^{\mathrm{TM}}$, Maplewood, MN), an empty guidewire casing with a preattached Luer-Lok ${ }^{\mathrm{TM}}$ (Beckton Dickinson, Inc., San Diego, CA) fitting, and a SureSeal ${ }^{\circledR}$ (Applied Medical, Inc., Rancho Santa Margarita, CA) adapter. The only

Department of Urology, University of California Irvine, Orange, California. 


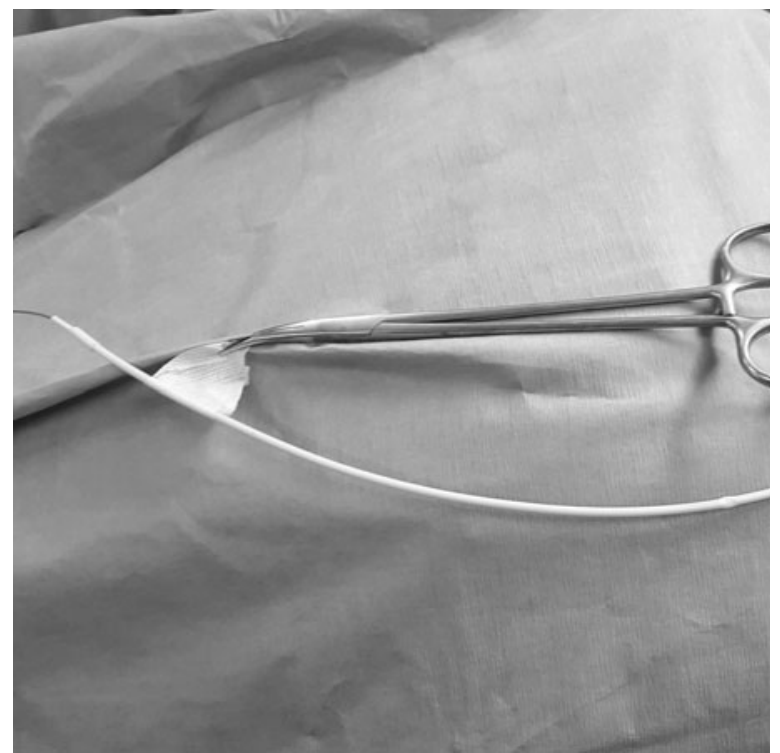

FIG. 3. A sterile adhesive strip wrapped around the yellow protective covering on the reusable fiber secured to the leg drape with a hemostat.

additional piece of equipment for this arrangement not routinely used during ureteroscopy is the sterile adhesive strip.

Step 1: Secure the optimal length of the laser fiber that needs to be in the operative field. Typically, this would be where the laser fiber drapes over the patient's leg. Place the sterile adhesive strip around the disposable fiber or around the yellow protective covering on the reusable fiber where it passes over the leg drape and, in turn, secures the sterile adhesive strip to the drape with a hemostat (Fig. 3).

Step 2: Pass the tip of the laser fiber through the leak-tight seal adapter (e.g., SureSeal, Applied Medical, Inc.) that is attached to the working port of the ureteroscope. Pass the laser fiber through the ureteroscope until the tip of the holmium laser fiber is just protruding outside the tip of the ureteroscope. This defines the functional length of the laser fiber.

Step 3: Detach the working port leak-tight adaptor from the ureteroscope, and without changing the position of the leak-tight adaptor on the holmium laser fiber, insert the laser fiber into one of the plastic casings that came with one of the guidewires. The chosen guidewire casing must have a preattached Luer-Lok (Beckton Dickinson, Inc.) fitting on one of its ends. Pass the laser fiber until the leak-tight adaptor is encountered and then secure it to the Luer-Lok (Beckton Dickinson, Inc.) fitting on the plastic guidewire casing. This will protect the laser fiber from accidental physical damage while maintaining its functional length (Fig. 4). The guidewire casing is secured to the drapes with two towel clips (Figs. 1 and 2).

At the time that the laser fiber is required for ureteroscopic lithotripsy, the leak-tight adaptor is twisted off of the LuerLok (Beckton Dickinson, Inc.) fitting on the guidewire casing

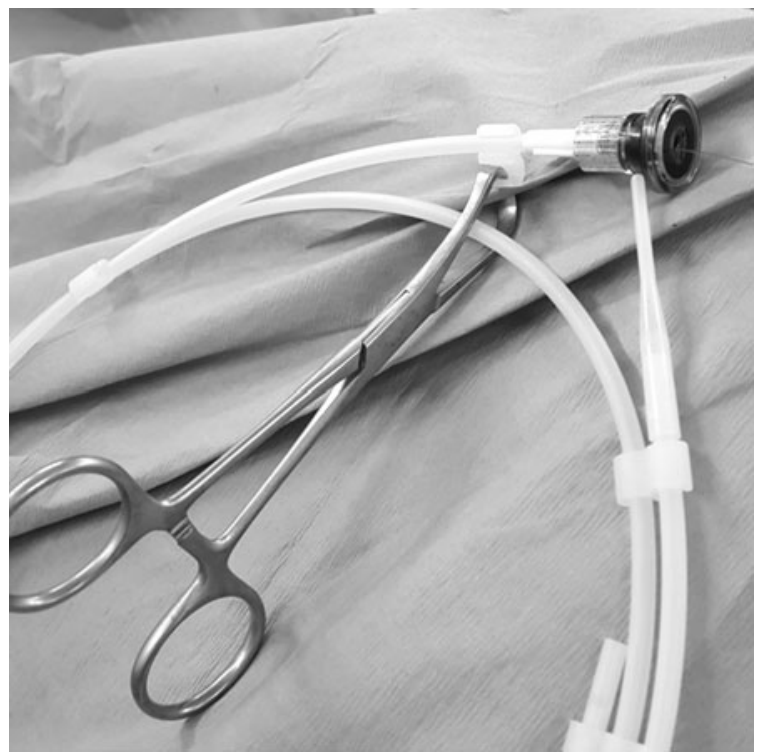

FIG. 4. A leak-tight adaptor on the laser fiber passed until it is connected to the Luer-Lok fitting on the plastic guidewire casing, ensuring proper storage at the appropriate functional length of the fiber.

and the holmium laser is removed from the guidewire casing all the time maintaining its functional length. The laser fiber is then passed into the ureteroscope until the leak-tight adaptor can be secured to the Luer-Lok (Beckton Dickinson, Inc.) fitting on the ureteroscope. At this point, the tip of the laser should be seen just exiting the tip of the ureteroscope and entering the surgical field. The laser fiber is safely and efficiently ready for use.

\section{Author Disclosure Statement}

No competing financial interests exist.

\section{References}

1. Stamatelou KK, Francis ME, Jones CA, et al. Time trends in reported prevalence of kidney stones in the United States: 1976-1994. Kidney Int 2003;63:1817-1823.

2. Matlaga BR. Contemporary surgical management of upper urinary tract calculi. J Urol 2009;181:2152-2156.

3. Chandhoke PS. Economics of urolithiasis: Cost-effectiveness of therapies. Curr Opin Urol 2001;11:391-393.

Address correspondence to:

Ryan A. Chandhoke, MD

Department of Urology

University of California Irvine

333 City Blvd. West, Ste. 2100

Orange, CA 92868

E-mail: rachandh@uci.edu 